\title{
Características morfológicas y limnológicas de las floraciones de Ceratium furcoides (Dinophyta) en un lago somero de Chile Central
}

\author{
Viviana Almanza ${ }^{1,2, *}$, Carlos E. de M. Bicudo ${ }^{3}$, Oscar Parra ${ }^{1,4}$ y Roberto Urrutia ${ }^{1,4}$ \\ ${ }^{1}$ Centro EULA-Chile, Universidad de Concepción, Barrio Universitario, Concepción, Chile. \\ ${ }^{2}$ Doctorado en Ciencias Ambientales, Facultad de Ciencias Ambientales, Universidad de Concepción, Concep- \\ ción, Chile. \\ ${ }^{3}$ Instituto de Botânica, Av. Miguel Estéfano, 3687, 04301-012 São Paulo, SP, Brasil. \\ ${ }^{4}$ Centro de Recursos Hídricos para la Agricultura y la Minería, Victoria 1295, Barrio Universitario, Concepción, \\ Chile.
}

* Corresponding author: valmanza@udec.cl

Received: 25/08/2015 Accepted: 13/02/2016

\begin{abstract}
RESUMEN
Características morfológicas y limnológicas de las floraciones de Ceratium furcoides (Dinophyta) en un lago somero de Chile central

Los dinoflagelados del género Ceratium forman floraciones no tóxicas en ecosistemas de agua continentales, que generan diversos efectos ecosistémicos, estéticos y en el abastecimiento del recurso hídrico. En este trabajo se describe por primera vez la presencia de la especie invasora Ceratium furcoides en Chile. Se recolectaron muestras mensuales entre enero del 2012 y diciembre del 2013 en el lago urbano de Lo Méndez, con el fin de estudiar la relación de la abundancia y morfología del dinoflagelado con las características limnológicas del cuerpo de agua. Los resultados demostraron elevada abundancia durante todo el año (promedio de $4440 \pm 3153$ ind./l) con variaciones estacionales, así como la formación de densas floraciones en la estación de otoño (máximo 19108 ind./1), las que se relacionaron principalmente con elevadas concentraciones de nitrógeno total, nitrato y amonio. Se observaron cambios en la morfología de $C$. furcoides principalmente de la longitud total y el número de cuernos de la hipoteca, que variaron dependiendo las condiciones ambientales y de su abundancia. Estos cambios morfológicos podrían constituir una ventaja competitiva en la colonización, permanencia y proliferación en ecosistemas hipereutróficos.
\end{abstract}

Palabras claves: Ceratium furcoides, floraciones, eutrofización, lagos urbanos, Chile.

\begin{abstract}
Limnological and morphological characteristics of Ceratium furcoides blooms (Dinophyta) in a shallow lake in central Chile

Dinoflagellates of the Ceratium genus form non-toxic blooms in freshwater ecosystems, generating diverse ecosystem, landscape and water supply effects. In this study, we report for the first time in Chile the presence of the invasive species Ceratium furcoides. Since January 2012 to December 2013 the species abundance and its morphology were studied in the urban lake Lo Méndez. Limnological characteristics of the lake were also described. Results showed high abundances throughout the year (average of $4440 \pm 3153$ ind./l) with seasonal changes and blooms in autumn (maximum 19108 ind./l), which were mainly associated with high concentrations of total nitrogen, nitrate, and ammonium. The morphology of $\mathrm{C}$. furcoides showed changes in the total length and number of horns of the hypotheca, which were related to the environmental conditions and its abundance. These morphological changes could constitute a competitive advantage to colonize, stay and grow in hypertrophic freshwater ecosystems.
\end{abstract}

Key words: Ceratium furcoides, blooms, eutrophication, urban lakes, Chile. 


\section{INTRODUCCIÓN}

Las especies del género Ceratium desarrollan floraciones en lagos y embalses meso- a hipereutróficos de zonas tropicales (Bustamante et al., 2012; Silva et al., 2012; Meichtry et al., 2014) y templadas (Donagh et al., 2005; Ginkel et al., 2007; Hart \& Wragg, 2009). Sus floraciones no son tóxicas, sin embargo, pueden modificar el color y sabor del agua, obstruir filtros en los sistemas de potabilización y generar la muerte de peces por el agotamiento del oxígeno disuelto cuando la población declina (Nicholls et al., 1980), lo que puede representar un serio problema ambiental y de uso del recurso hídrico (Hart \& Wragg, 2009; Silverio et al., 2009; Matsumura-Tundisi et al., 2010).

El género Ceratium fue típicamente registrado en el hemisferio norte, y con mucha menos frecuencia en el hemisferio sur (Margalef, 1983). Sin embargo, en los últimos años se han realizado diferentes registros en Sudáfrica (Ginkel et al., 2007; Hart \& Wragg, 2009) y en Suramérica (Donagh et al., 2005; Silverio et al., 2009; Meichtry et al., 2014). Para el caso de los lagos Andino Patagónicos de Argentina la cita más antigua corresponde a Ceratium hirundinella (O.F. Müller) Schrank (Thomasson, 1963). Esta misma especie fue descrita para el sur de Chile (Parra, 1998). Actualmente, se han realizado continuas citas de otra especie del género Ceratium, $C$. furcoides (Levander) Langhans 1925, de la que no se tenía conocimiento en la región hasta recientes observaciones en Argentina (Meichtry et al., 2014) y Brasil (Santos-Wisniewski et al., 2007; Oliveira et al., 2011; Silva et al., 2012; Pereira et al., 2013). Esta especie es morfológicamente muy similar a $C$. hirundinella, por lo que probablemente han sido documentadas como una sola en el pasado (Calado \& Larsen, 1997). Tanto $C$. hirundinella como $C$. furcoides, han sido reconocidas como invasoras en ambientes dulceacuícolas en Suramérica (Silva et al., 2012; Pereira et al., 2013; Meichtry et al., 2014).

La rápida dispersión y establecimiento del genero Ceratium en Suramérica se puede asociar a rasgos que se consideran comunes en los organismos invasores: alta tasa de crecimiento, alta efi- ciencia en la utilización de recursos y capacidades competitivas superiores sobre las especies nativas cuando las condiciones locales varían ( $\mathrm{Su}-$ kenik et al., 2012). El gran tamaño de las especies de Ceratium evita la depredación del zooplancton, la presencia de flagelos les permite hacer migraciones a estratos profundos ricos en nutrientes en distintos momentos del día, así como cambiar su distribución con respecto a los gradientes de luz. Con la formación de quistes que sedimentan ante condiciones ambientales desfavorables logran permanecer por largos periodos de tiempo en un cuerpo de agua (Pollingher, 1988; Reynolds, 2006). Además, Ceratium puede presentar diferentes morfologías asociadas a variaciones en los factores ambientales así como al ciclo de vida (cliclomorfosis) (Lindström, 1992; Gligora et al., 2003; Parodi et al., 2007). En C. furcoides se ha descrito la variabilidad morfológica en una población del lago Erken, Suecia (Lindström, 1992), sin información en hábitats donde ha ocurrido su reciente invasión. En este contexto, se ha planteado la hipótesis que el establecimiento de $C$. furcoides y el desarrollo de floraciones en el lago Lo Méndez está relacionado con la eutrofización del sistema, de modo que aumentos de abundancia junto con cambios morfológicos externos favorecen su dominancia y establecimiento en el lago.

El objetivo del presente estudio fue determinar los principales factores que influyen en el desarrollo y la proliferación de $C$. furcoides en el lago eutrófico de Lo Méndez, estableciendo si la densidad y morfología se relacionan con los cambios abióticos y bióticos estacionales típicos de sistemas de la zona templada.

\section{MATERIALES Y MÉTODOS}

\section{Área de estudio}

El lago Lo Méndez $\left(36^{\circ} 48^{\prime} 03^{\prime \prime} \mathrm{S}\right.$ y $\left.73^{\circ} 03^{\prime} 03^{\prime \prime} \mathrm{O}\right)$ está ubicado en la ciudad de Concepción, Región del Biobío, Chile central (Fig. 1). Es un lago somero (profundidad máxima de $6.5 \mathrm{~m}$ ), tiene un área de $4.2 \mathrm{~km}^{2}$ y un volumen promedio de $157800 \mathrm{~m}^{3}$, no presenta estratificación térmi- 
ca en verano. Por la elevada concentración de nutrientes en la columna de agua fue clasificado como hipereutrófico (Andrés, 2012). Forma parte del sistema de lagos urbanos de Concepción, por lo que constituye un importante espacio público de valor paisajístico y recreativo para sus habitantes. Además, es considerado como un reservorio de agua para la ciudad ante un eventual desabastecimiento del recurso.

\section{Diseño de muestreo}

En el año 2011 se realizó un pre muestreo, que consistió en la toma de datos de diferentes variables físico-químicas y de muestras para el estudio de la comunidad de fitoplancton en diferentes sitios del lago y profundidades (superficie y fondo) durante las cuatro estaciones del año. Los resultados del análisis de conglomerados y ANOSIM

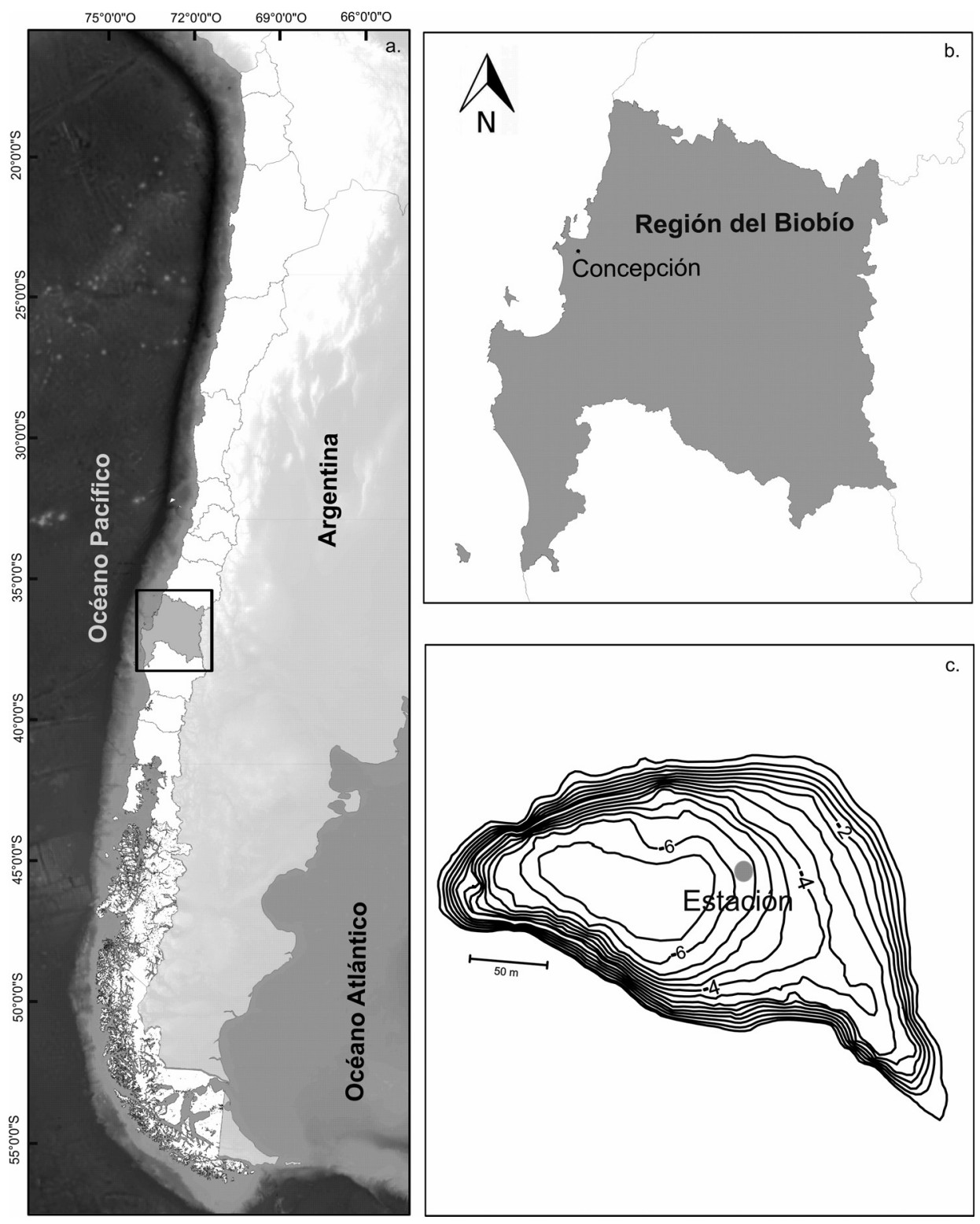

Figura 1. Área de estudio, Lago urbano de Lo Méndez. a. Chile. b. Región del Biobío. c. Batimetría del lago. Study area, Lo Méndez urban lake. a. Chile, b. Biobío region. c. Bathymetry. 
a dos vías (factores sitio de muestreo y profundidad) mostraron que no existían diferencias significativas entre los sitios de muestreo y en las diferentes profundidades, por lo que para el presente estudio se tomaron muestras integradas en un solo sitio de muestreo, con una periodicidad mensual desde enero del 2012 a diciembre del 2013 (Fig. 1).

\section{Registro de variables físico-químicas en la columna de agua}

Las variables físico-químicas de calidad del agua como temperatura $\left({ }^{\circ} \mathrm{C}\right), \mathrm{pH}$, conductivi$\mathrm{dad}(\mu \mathrm{S} / \mathrm{cm})$ y oxígeno disuelto $(\mathrm{mg} / \mathrm{l})$ fueron medidas in situ en la columna del agua (a 20 $\mathrm{cm}, 2 \mathrm{~m}$ y $4 \mathrm{~m}$ ) con una sonda multiparámetrica (Hydrolab modelo Quanta). La transparencia del agua se estimó como la profundidad de visión del disco Secchi $(\mathrm{cm})$.

Las muestras de agua fueron tomadas con una botella Niskin vertical de 1.51 de volumen en dos profundidades (superficie y fondo). Posteriormente se mezclaron en un recipiente plástico y se obtuvo una muestra integrada de la columna de agua. Las muestras de agua se trasladaron en condiciones de frío $\left(4^{\circ} \mathrm{C}\right)$ y oscuridad a diferentes laboratorios para el análisis de la comunidad de fitoplancton, concentración de clorofila $a$ $\mathrm{y}$ análisis de nutrientes.

\section{Análisis químico de muestras de agua}

Las muestras fueron analizadas por espectrofotometría de absorción molecular y/o cromatografía iónica según procedimientos descritos en APHA (2012); fósforo total (PT) (4500 P), ortofosfato $\left(\mathrm{PO}_{4}^{3-}\right)(4110 \mathrm{P})$, nitrógeno total (NT) (4500$\mathrm{N} \mathrm{C})$, nitrato $\left(\mathrm{NO}_{3}^{-}\right)(4110-\mathrm{NO} 3)$, nitrito $\left(\mathrm{NO}_{2}^{-}\right)$ (4110-NO2) y amonio $\left(\mathrm{NH}_{4}^{+}\right)$(4500-NH3).

\section{Análisis de la comunidad fitoplanctónica e identificación taxonómica de Ceratium furcoides}

Las muestras de fitoplancton fueron fijadas in situ con solución lugol al $1 \%$. Una vez en el laboratorio, se cuantificó el número de células de microalgas por el método de Utermöhl (1958), utilizando cámaras de sedimentación de volumen conocido (5 y $10 \mathrm{ml}$ dependiendo la muestra) en un microscopio invertido marca Zeiss Axiovert 35. La identificación taxonómica se realizó hasta en nivel de género y/o especie siguiendo la clasificación a nivel de clase propuesto por Pascher (1931-1941).

La identificación taxonómica de $C$. furcoides se llevó a cabo sobre individuos recolectados durante cada uno de los muestreos mensuales con el fin de diferenciar esta especie de $C$. hirundinella. Las diferencias descritas para $C$. hirundinella y $C$. furcoides se basan en el aspecto de la base de la epiteca, que es acampanada en $C$. hirundinella y casi cónica en $C$. furcoides (Calado \& Larsen, 1997). Las cuatro placas del cuerno apical se extienden hasta el ápice en $C$. hirundinella, mientras que en $C$. furcoides la cuarta placa es de menor tamaño y no alcanza el ápice (Popovský \& Pfiester, 1990; Calado \& Larsen, 1997; Pereira et al., 2013). Para identificar estas diferencias entre las dos especies fue necesario separar las placas de los dinoflagelados, aplicando hipoclorito de sodio $5 \%$ bajo el cubreobjetos y observando las placas de la epiteca al microscopio fotónico (Boltovskoy, 1989). Para confirmar la configuración y tamaño de las mismas se analizaron muestras de diferentes meses con un microscopio electrónico de barrido (SEM) siguiendo las especificaciones de Carty (2003).

Las variaciones en la morfología del dinoflagelado fueron determinadas a partir de mediciones en un mínimo de 15 individuos escogidos al azar de cada muestra mensual. Se utilizó un microscopio Carl Zeiss @Axioplan con el software Zen lite 2012. Se midieron 5 caracteres morfológicos: longitud total del individuo (LT), ancho de la epiteca en el cíngulo (c), largo del cuerno de la epiteca (h1), largo (Z) y base de los cuernos de la hipoteca (d). También se enumeraron los cuernos presentes en la hipoteca. Todas las medidas fueron realizadas de acuerdo a Vadrucci et al. (2007) para la estimación del biovolumen de Ceratium.

Para la determinación de la concentración de clorofila $a$ se filtraron $250 \mathrm{ml}$ de muestra a través de filtros de membrada de celulosa $(0.45 \mu \mathrm{m})$. Una vez terminada la filtración, el filtro fue in- 
troducido en un frasco plástico oscuro con $20 \mathrm{ml}$ de acetona al $90 \%$ durante 24 horas para la extracción total del pigmento. La determinación de la concentración de clorofila $a$ se realizó con un fluorómetro 10-AU Turner Designs.

\section{Análisis estadísticos}

Para caracterizar las condiciones limnológicas donde se desarrollaron las poblaciones de $C$. furcoides se realizó un análisis de componentes principales (PCA) en base a las variables físicoquímicas registradas estacionalmente (verano, otoño, invierno y primavera) durante cada año estudiado. Para ello, se estimó previamente el valor promedio característico para cada época y año. Las diferencias significativas entre los periodos y años fueron determinadas con un análisis ANOSIM de dos vías. Los datos de variables físico-químicas y de abundancia de $C$. furcoides fueron transformados con $\log (x+1)$ para su normalización. Mediante una regresión lineal múltiple, se identificó cuál de estos factores influyó en la abundancia y longitud total de $C$. furcoides. Una correlación de Pearson permitió determinar asociaciones significativas entre la abundancia de $C$. furcoides y las características limnológicas estacionales anuales.

Se utilizó un análisis ANOVA de una vía $(p<$ $0.05)$ para determinar la significancia de las diferencias entre variables físico-químicas, abundancia y medidas morfométricas de $C$. furcoides. La

Tabla 1. Variables limnológicas e índices comunitarios en el lago Lo Méndez: temperatura (Temp.), transparencia (Transp.), Conductividad (Cond.), $\mathrm{pH}$, oxígeno disuelto (OD), nitrógeno total $(\mathrm{NT})$, nitratos $\left(\mathrm{NO}_{3}^{-}\right)$, nitritos $\left(\mathrm{NO}_{2}^{-}\right)$, amonio $\left(\mathrm{NH}_{4}^{+}\right)$, fósforo total (PT), ortofosfatos $\left(\mathrm{PO}_{4}^{3-}\right)$, clorofila $a$, riqueza de especies $(\mathrm{S})$ y índice de diversidad de Shannon $\left(H_{\log _{10}}^{\prime}\right)$ (promedio \pm desviación estándar DS), <al límite de detección. Según Vollenweider \& Kerekes (1982): HI = Hipereutrófico. Limnology parameters and community descriptors in Lo Mendez lake: temperature (Temp.), transparency (Transp.), Conductivity (Cond.), pH, dissolved oxygen $(\mathrm{DO})$, total nitrogen $(\mathrm{NT})$, nitrate $\left(\mathrm{NO}_{3}^{-}\right)$, nitrite $\left(\mathrm{NO}_{2}^{-}\right)$, ammonium $\left(\mathrm{NH}_{4}^{+}\right)$, total phosphorus $(\mathrm{PT})$, Orthophosphate $\left(\mathrm{PO}_{4}^{3-}\right)$, chlorophyll a, species richness $(S)$ and Shannon diversity index $\left(\mathrm{H}_{\log _{10}}^{\prime}\right)($ mean \pm standard deviation $S D,<$ detection limit. According to Vollenweider \& Kerekes (1982): HI = Hypertrophic.

\begin{tabular}{|c|c|c|c|c|c|c|c|c|c|}
\hline Variable & $\begin{array}{c}\text { Verano } \\
2012\end{array}$ & $\begin{array}{l}\text { Otoño } \\
2012\end{array}$ & $\begin{array}{c}\text { Invierno } \\
2012\end{array}$ & $\begin{array}{c}\text { Primavera } \\
2012\end{array}$ & $\begin{array}{c}\text { Verano } \\
2013\end{array}$ & $\begin{array}{c}\text { Otoño } \\
2013\end{array}$ & $\begin{array}{c}\text { Invierno } \\
2013\end{array}$ & $\begin{array}{c}\text { Primavera } \\
2013\end{array}$ & Total \\
\hline $\mathrm{mp} .\left({ }^{\circ} \mathrm{C}\right)$ & $22.6 \pm 1.5$ & $14.3 \pm 3.8$ & $13.6 \pm$ & & $22.4 \pm$ & & & & 17.3 \\
\hline Transp. (m) & $0.8 \pm 0.0$ & $0.8 \pm 0.1$ & $1.0 \pm 0.6$ & & $1.4 \pm 0.5$ & & & $1.4 \pm 0.6$ & $1.2 \pm 0.7(\mathrm{HI})$ \\
\hline $\begin{array}{l}\text { Cond. } \\
(\mu \mathrm{m} / \mathrm{cm})\end{array}$ & $277.8 \pm 17.6$ & $257.2 \pm 29.6$ & $223.0 \pm 7.0$ & $210.6 \pm 45.7$ & $261.7 \pm 21.5$ & $276.3 \pm 15.3$ & $253.6 \pm 19.9$ & $223.7 \pm 11.7$ & $248.0 \pm 31.6$ \\
\hline $\mathrm{pH}$ & $7.7 \pm 0.2$ & $7.2 \pm 0.3$ & $7.3 \pm 0.1$ & $7.4 \pm 0.1$ & $8.0 \pm 0.3$ & $7.9 \pm 0.6$ & $7.5 \pm 0.6$ & $8.2 \pm 0.5$ & $7.7 \pm 0.5$ \\
\hline $\mathrm{OD}(\mathrm{mg} / \mathrm{l})$ & $8.3 \pm 1.9$ & $9.2 \pm 0.8$ & $9.3 \pm 1.1$ & $6.8 \pm 0.4$ & $8.2 \pm 2.3$ & $8.7 \pm 0.9$ & $7.6 \pm 0.2$ & $7.5 \pm 1.5$ & $8.2 \pm 1.4$ \\
\hline NT (mg/l) & $2.1 \pm 0.4$ & $1.4 \pm 0.5$ & $1.4 \pm 0.2$ & $1.0 \pm 0.3$ & $0.8 \pm 0.1$ & $1.2 \pm 0.2$ & $1.9 \pm 0.1$ & $0.7 \pm 0.3$ & $1.3 \pm 0.5$ \\
\hline $\mathrm{NO}_{3}^{-}(\mathrm{mg} / \mathrm{l})$ & $1.43 \pm 0.2$ & $0.93 \pm 0.5$ & $1.40 \pm 0.4$ & $0.37 \pm 0.3$ & $<0.02$ & $<0.02$ & $1.10 \pm 0.3$ & $0.04 \pm 0.0$ & $0.66 \pm 0.6$ \\
\hline $\mathrm{NO}_{2}^{-}(\mathrm{mg} / \mathrm{l})$ & $<0.02$ & $<0.02$ & $0.06 \pm 0.02$ & $<0.02$ & $0.02 \pm 0.01$ & $<0.02$ & $<0.02$ & $0.02 \pm 0.01$ & $0.02 \pm 0.02$ \\
\hline $\mathrm{NH}_{4}^{+}(\mathrm{mg} / \mathrm{l})$ & $0.89 \pm 0.35$ & $0.26 \pm 0.40$ & $0.33 \pm 0.10$ & $0.28 \pm 0.17$ & $0.04 \pm 0.01$ & $0.04 \pm 0.01$ & $0.06 \pm 0.03$ & $0.08 \pm 0.10$ & $0.25 \pm 0.32$ \\
\hline PT (mg/l) & $0.06 \pm 0.00$ & $0.09 \pm 0.02$ & $0.10 \pm 0.01$ & $0.07 \pm 0.01$ & $0.08 \pm 0.03$ & $0.11 \pm 0.04$ & $0.10 \pm 0.05$ & $0.10 \pm 0.08$ & $0.09 \pm 0.04(\mathrm{HI})$ \\
\hline $\mathrm{PO}_{4}^{3-}(\mathrm{mg} / \mathrm{l})$ & $0.98 \pm 0.03$ & $0.25 \pm 0.37$ & $<0.04$ & $<0.04$ & $<0.04$ & $<0.04$ & $<0.04$ & $<0.04$ & $0.18 \pm 0.33$ \\
\hline $\begin{array}{l}\text { Clorofila } a \\
(\mu \mathrm{g} / \mathrm{l})\end{array}$ & $100.4 \pm 23.4$ & $78.4 \pm 57.7$ & $20.5 \pm 8.8$ & $13.9 \pm 6.0$ & $20.5 \pm 1.4$ & $110.5 \pm 6.5$ & $73.2 \pm 47.4$ & $24.6 \pm 4.4$ & $55.3 \pm 44.6(\mathrm{HI})$ \\
\hline S & $11 \pm 1$ & $13 \pm 4$ & $12 \pm 4$ & $17 \pm 6$ & $20 \pm 5$ & $15 \pm 5$ & $16 \pm 4$ & $19 \pm 7$ & $15 \pm 5$ \\
\hline$H^{\prime}\left(\log _{10}\right)$ & $0.7 \pm 0.1$ & $0.8 \pm 0.1$ & $0.4 \pm 0.1$ & $0.8 \pm 0.2$ & $0.8 \pm 0.1$ & $0.7 \pm 0.1$ & $0.5 \pm 0.1$ & $0.8 \pm 0.2$ & $0.7 \pm 0.2$ \\
\hline
\end{tabular}


relación entre la longitud total y la abundancia del dinoflagelado fue explicada mediante una regresión lineal simple. El análisis de varianza también se empleó para determinar diferencias entre la abundancia, riqueza y diversidad de taxa (índices comunitarios) por estación y por año, e identificadas con la prueba post hoc de Tukey. Todos los análisis fueron llevados a cabo utilizando los programas R versión 2.14.1 y Primer 6.0.

\section{RESULTADOS}

\section{Factores abióticos y abundancia de Ceratium furcoides}

El lago urbano de Lo Méndez presentó aguas neutras a alcalinas, de alta conductividad eléctri- ca y alta concentración de nutrientes (Tabla 1). Atendiendo a sus valores promedios de transparencia del agua $(1.2 \mathrm{~m})$, concentración de PT $(0.09 \mathrm{mg} / \mathrm{l})$ y de clorofila $a(55.3 \mu \mathrm{g} / \mathrm{l})$, el lago presentó un estado hipereutrófico (Vollenweider \& Kerekes, 1982), que se mantuvo durante todas las estaciones del año.

El Análisis de Componentes Principales PCA (Fig. 2) indicó que la concentración de PT, NT, $\mathrm{NO}_{2}^{-}$, temperatura, oxígeno disuelto, transparencia del agua y conductividad eléctrica son las variables que mejor explican las condiciones limnológicas en Lo Méndez, con un 56.9\% de la varianza entre los dos primeros ejes. Variaciones estacionales en la concentración de nutrientes y en la temperatura $(F=22.09$; g.1. $3 ; p<0.05)$ determinaron diferencias estacionales en las condiciones del agua, principalmente entre las esta-

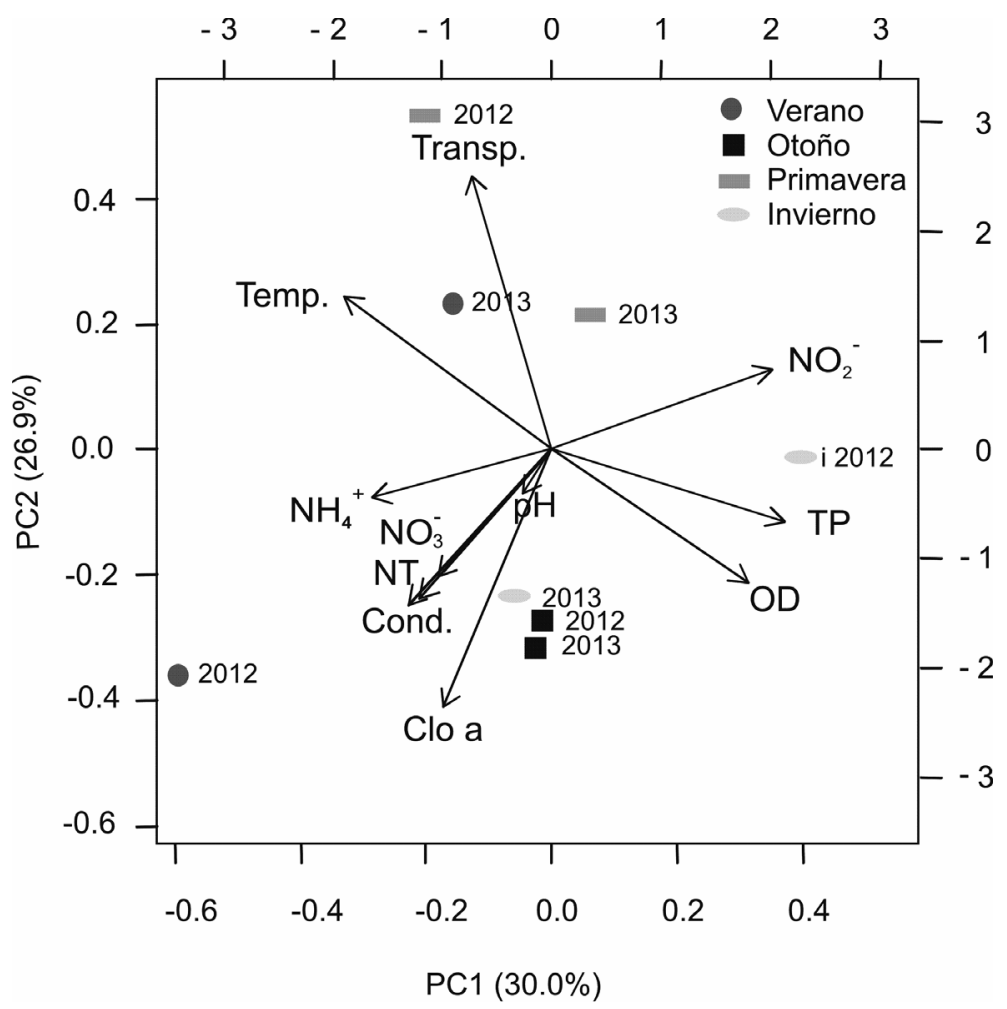

Figura 2. Diagrama de ordenación PCA de las variables físicas y químicas más importantes en el lago urbano de Lo Méndez asociados a las diferentes épocas del año, valores promedio por estación por año de estudio. Temp. (Temperatura), Transp.

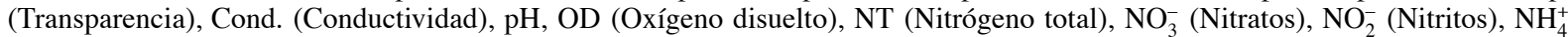
(Amonio), PT (Fósforo total), Clo a (Clorofila a). PCA ordination diagram correlating the most significant physical and chemical variables measured in Lo Méndez lake. Temp. (temperature), Transp. (transparency), Cond. (Conductivity), pH, OD (Dissolved oxygen), $\mathrm{NT}$ (Total nitrogen), $\mathrm{NO}_{3}^{-}$(Nitrate), $\mathrm{NO}_{2}^{-}$(Nitrite), $\mathrm{NH}_{4}^{+}$(Ammonium), PT (Total phosphorus), Clo a (Chlorophyll a). 
ciones de otoño-invierno en relación a primaveraverano (ANOSIM $R_{\text {global }}=0.57 ; p<0.001$ ). Estas diferencias no fueron evidentes entre los años estudiados (ANOSIM $\left.R_{\text {global }}=0.53 ; p>0.05\right)$.

Los resultados del análisis de regresión lineal múltiple demostraron que la concentración $\mathrm{NO}_{3}^{-}$ influyó positivamente en la abundancia del dinoflagelado (Tabla 2).

Durante la estación de otoño del año 2012 y 2013 se presentaron floraciones del dinoflagelado C. furcoides. Su abundancia se correlacionó positivamente con las concentraciones de $\mathrm{NT}, \mathrm{NH}_{4}^{+} \mathrm{y}$ $\mathrm{PO}_{4}^{3-}$ en esta época (Tabla 3). Durante el desarrollo de la floración fue registrada una menor transparencia del agua con respecto a otras épocas del año $(F=4.02$; g.1. $3 ; p<0.05)$, especialmente si se compara con la primavera, así como un aumento de la concentración de la clorofila $a(F=2.02$; g.l. $3 ; p>0,05)$ y de la concentración de oxígeno disuelto $(F=2.62$; g.1. 3; $p>0.05)$. Sin embargo, estas diferencias no fueron estadísticamente significativas (Tabla 1). Las proliferaciones se desarrollaron en un rango de temperatura que varió entre 12 y $19{ }^{\circ} \mathrm{C}$.

\section{Factores bióticos y abundancia de Ceratium furcoides}

Se identificaron 44 taxa de fitoplancton en el lago durante el periodo de muestreo, que pertenecen a siete clases: Chlorophyceae (18 taxa), Bacillariophyceae (13 taxa), Cyanobacteria (4 taxa), Dinophyceae (3 taxa) Euglenophyceae (3 taxa), Cryptophyceae (2 taxa) y Chysophyceae (1 taxa) (Fig. 3). Los dinoflagelados (Ceratium furcoides, Peridinium sp. y Peridiniopsis elpatiewskyi), las diatomeas (Asterionella formosa y Fragilaria crotonensis), y las algas verdes (Closterium aciculare y Desmodesmus communis) dominaron la comunidad $(39 \%, 29 \%$ y $17 \%$ respectivamente). Las otras clases de fitoplancton estuvieron presentes en baja abundancia $(<16 \%$ del total del fitoplancton).

C. furcoides mantuvo una alta abundancia durante todo el año (promedio de $4440 \pm 3153$ ind./l) y presentó un ciclo anual estacional, según el cual su abundancia fue significativamente menor durante la primavera $(2012=2029 \pm 768$ ind./1 y $2013=2216 \pm 1721$ ind./l) y mayor du-

Tabla 2. Regresión lineal múltiple de la abundancia y longitud total de Ceratium furcoides. Multiple linear regression of abundance and total length of Ceratium furcoides.

\begin{tabular}{|c|c|c|c|c|}
\hline & Coeficiente & Error estándar & Estad. & Valor- $p$ \\
\hline \multicolumn{5}{|c|}{ Abundancia de $C$. furcoides } \\
\hline Constante & 11646.82 & 4134.50 & 2.817 & $0.01 *$ \\
\hline Nitrógeno total (NT) & -5220.79 & 2188.97 & -2.38 & $0.03 *$ \\
\hline Nitratos $\left(\mathrm{NO}_{3}^{-}\right)$ & 3444.05 & 1170.38 & 2.94 & $0.01 *$ \\
\hline Nitritos $\left(\mathrm{NO}_{2}^{-}\right)$ & -44827.79 & 31804.74 & -1.40 & 0.18 \\
\hline Amonio $\left(\mathrm{NH}_{4}^{+}\right)$ & -141.49 & 1955.36 & -2.62 & $0.02 *$ \\
\hline Clorofila $a$ & 36.75 & 12.00 & 3.06 & $0.01 *$ \\
\hline Índice de diversidad $\left(H^{\prime} \log _{10}\right)$ & -6704.04 & 4273.11 & -1.56 & 0.14 \\
\hline \multicolumn{5}{|c|}{ Longitud total de $C$. furcoides } \\
\hline Constante & 159.70 & 17.91 & 8.91 & $0.00 *$ \\
\hline Nitrógeno total (NT) & -23.96 & 13.72 & -1.75 & 0.10 \\
\hline Nitratos $\left(\mathrm{NO}_{3}^{-}\right)$ & 24.22 & 12.05 & 2.01 & 0.06 \\
\hline Nitritos $\left(\mathrm{NO}_{2}^{-}\right)$ & 1065.81 & 516.66 & 2.06 & $0.05 *$ \\
\hline Amonio $\left(\mathrm{NH}_{4}^{+}\right)$ & 52.93 & 19.77 & 2.68 & $0.02 *$ \\
\hline
\end{tabular}




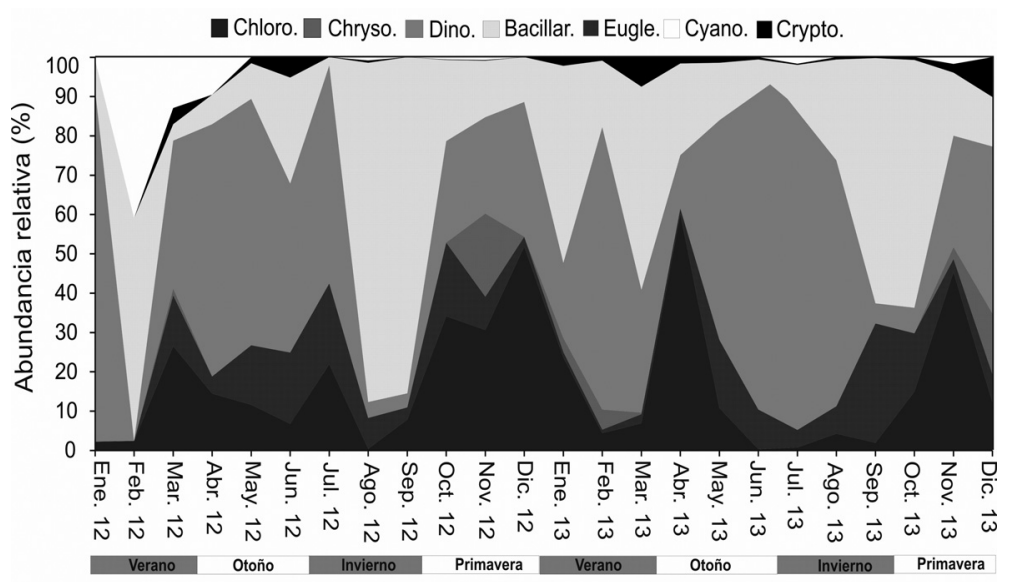

Figura 3. Abundancia relativa de los diferentes grupos que componen la comunidad de fitoplancton (Chloro: Chlorophyceae, Chryso: Chrysophyceae, Dino: Dinophyceae, Bacillar: Bacillariophyceae, Eugle: Euglenophyceae, Cyano: Cyanobacteria, Crypto: Cryptophyceae). Relative abundance of groups that make up the phytoplankton community (Chloro: Chlorophyceae, Chryso: Chrysophyceae, Dino: Dinophyceae, Bacillar: Bacillariophyceae, Eugle: Euglenophyceae, Cyano: Cyanobacteria, Crypto: Cryptophyceae).

rante el otoño de los dos años estudiados, con 19108 ind./l en abril del 2012 y 16482 ind./l a principios de junio del 2013 ( $F=46.4$; g.l. $7 ; p<0.05)$, correspondiendo con densas floraciones (Fig. 4).

Durante y después de las floraciones de $C$. furcoides la riqueza y diversidad de taxa fueron menores (otoño e invierno) en comparación con las otras estaciones del año (como en primavera, cuando se observó un aumento de la abundancia relativa de diatomeas y algas verdes). Estas dife- rencias fueron significativas sólo para la diversi$\operatorname{dad}\left(H^{\prime} \log _{10}\right)(F=7.1 ;$ g.l. $3 ; p<0.05)$.

\section{Presencia y morfología de Ceratium furcoides}

La identificación taxonómica mediante la morfología y el reconocimiento de la cuarta placa apical de los individuos colectados confirmó la presencia de $C$. furcoides en el lago Lo Méndez (Fig. 5). Las células, comprimidas dorso ventralmente mostraron un promedio de $47.7 \pm 9.9 \mu \mathrm{m}$

Tabla 3. Correlación de Pearson entre la abundancia de C. furcoides y las variables limnológicas. Pearson correlation between the abundance of $\mathrm{C}$. furcoides and limnological variables.

\begin{tabular}{|c|c|c|c|c|}
\hline Variable & Verano & Otoño & Invierno & Primavera \\
\hline Temperatura $(\mathrm{T})$ & -0.14 & 0.23 & -0.81 & 0.77 \\
\hline Transparencia (Transp.) & 0.55 & 0.18 & -0.48 & 0.12 \\
\hline Conductividad (Cond.) & -0.19 & -0.16 & 0.14 & 0.11 \\
\hline $\mathrm{pH}$ & 0.71 & 0.40 & 0.92 & 0.41 \\
\hline Oxígeno disuelto (OD) & 0.26 & -0.01 & -0.18 & -0.23 \\
\hline Nitrógeno total (NT) & -0.72 & 0.68 & 0.14 & -0.50 \\
\hline Nitratos $\left(\mathrm{NO}_{3}^{-}\right)$ & -0.90 & 0.41 & -0.54 & -0.08 \\
\hline Nitritos $\left(\mathrm{NO}_{2}^{-}\right)$ & 0.71 & -0.69 & -0.55 & -0.57 \\
\hline Amonio $\left(\mathrm{NH}_{4}^{+}\right)$ & -0.80 & 0.68 & -0.66 & -0.39 \\
\hline Fósforo total (PT) & 0.64 & -0.04 & 0.85 & -0.45 \\
\hline Orto fosfato $\left(\mathrm{PO}_{4}^{3-}\right)$ & -0.88 & 0.69 & 0.45 & -0.22 \\
\hline Clorofila $a$ (Clo. a) & -0.40 & 0.17 & 0.89 & 0.11 \\
\hline
\end{tabular}


de ancho y una longitud total que varió entre 109.9 a $311.5 \mu \mathrm{m}$ (promedio $169.7 \pm 32.1 \mu \mathrm{m}$ ) (Tabla 4). Las cuatro placas de la epiteca forman un cuerno largo y estrecho, mientras que la hipoteca, ancha y corta, está formada por dos o tres cuernos.

Los individuos presentaron variaciones morfológicas principalmente en la longitud total $(F=$ 20.6 ; g.1. $7 ; p<0.05)$ y en el número de cuernos de la hipoteca $(F=9.3$; g.1. $7 ; p<0.05)$, que variaron dependiendo la estación del año. La longitud total fue significativamente menor en otoño del $2013(145.2 \pm 10.1 \mu \mathrm{m})$. El mayor valor promedio de longitud se presentó en verano 2012 (200.0 $\pm 63.1 \mu \mathrm{m})$ (Fig. 4). Durante esta estación, la longitud del cuerno de la epíteca (h1) $(F=29.5 ;$ g.1. $7 ; p<0.05)$, la base (d) $(F=12.2$; g.1. $7 ; p<0.05)$, la longitud total de los cuernos $(Z)(F=21.9 ;$ g.1. $7 ; p<0.05)$ y el ancho de la epiteca (c) $(F=34$; g.1. $7 ; p<0.05)$, también fueron significativamente diferentes, y predominaron individuos con dos cuernos antapicales. En contraste con la primavera (2012 y 2013), cuando se identificó un mayor porcentaje de individuos con tres cuernos antapicales y de mayor tamaño (Tabla 4).

Cuando aumentó la abundancia, los individuos de $C$. furcoides presentaron una menor lon- gitud total, como demuestran los datos de otoño del 2012 y 2013 (11 $257 \pm 7050$ ind $/ 1$ y 7807 $\pm 7542 \mathrm{ind} / 1 \mathrm{respectivamente)}$, verano del 2013 (4666 \pm 1473 ind/l) e invierno del 2013 (4413 \pm 3359 ind./1). Por el contrario, cuando la abundancia fue menor, incrementó el tamaño de $C$. furcoides, existiendo una correlación negativa entre la longitud total y la abundancia. La concentración de $\mathrm{NH}_{4}^{+}$y $\mathrm{NO}_{2}^{-}$parece influir sobre una mayor longitud total del dinoflagelado (Tabla 2).

\section{DISCUSIÓN}

En este trabajo se describe por primera vez la presencia de la especie invasora Ceratium furcoides en Chile. La descripción morfológica, así como las medidas morfométricas de los individuos fueron consistentes con registros anteriores para la especie (Popovský \& Pfiester, 1990; Meichtry et al., 2014). Hasta el momento se habían descrito en el país dos especies del género Ceratium: C. tetraceras Schrank 1841 y C. hirundinella (Parra, 2006). Este último habría sido introducido en la década de los 80 por el traslado de quistes del dinoflagelado junto con ovas de salmón durante el progreso de la salmonicultura en el sur de Chile (Parra, 1998). Sin embar-

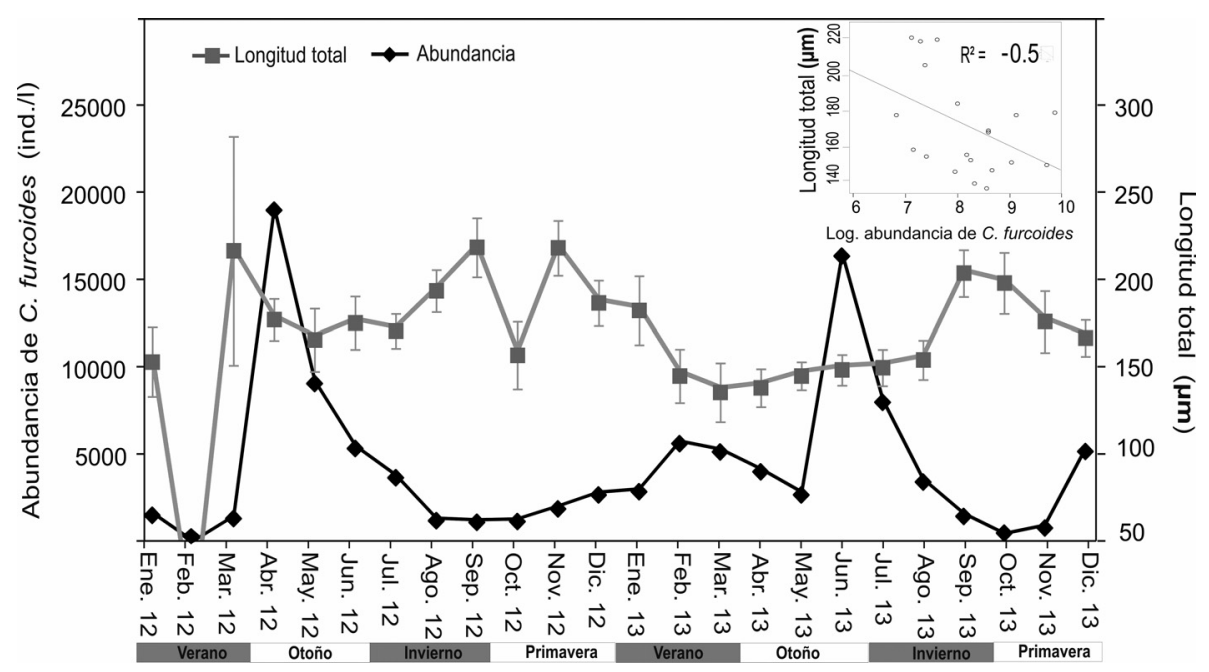

Figura 4. Abundancia y longitud total de C. furcoides en el lago Lo Méndez. Regresión lineal de la longitud total de $C$. furcoides en relación con su abundancia. Total abundance and length of $\mathrm{C}$. furcoides in Lo Mendez. Lake. Linear regression of total length of C. furcoides in relation to their abundance. 
go, no existen otros antecedentes que den cuenta de la introducción y dispersión de esta especie en el país. De la misma forma, el proceso de colonización de $C$. furcoides en Suramérica no está del todo clara, se ha asociado a cambios climáticos, a modificaciones en el régimen hidrológico, a cambios en la calidad del agua y al transporte de estados de resistencia (quistes) por aves, insectos acuáticos o por intervención antrópica (Parra, 1998; Pereira et al., 2013; Meichtry et al., 2014). El registro de $C$. furcoides en el lago urbano de Lo Méndez da cuenta de su rápida dispersión por Suramérica y del establecimiento en lagos eutróficos, como se ha descrito en otras zonas de la región (Silva et al., 2012; Pereira et al., 2013; Meichtry et al., 2014). Aunque probablemente se encuentre en otros ecosistemas acuáticos con similares características de concentración de nutrientes. El desconocimiento de su presencia en Chile puede haber contribuido a la subestimación de su área de distribución y a identificaciones erróneas como $C$. hirundinella, considerando su similar morfología, requerimientos ambientales (Bustamante et al., 2012) y coexis- tencia en el mismo cuerpo de agua (Heaney et al., 1988; Lindström, 1992). Nuestros resultados muestran que $C$. furcoides y $C$. hirundinella no coexisten en el lago Lo Méndez, sin embargo, este último ha sido registrado en varios lagos del centro y sur del país (Parra, 2006).

La alta concentración de nutrientes en el lago urbano de Lo Méndez favoreció la presencia del dinoflagelado en concordancia con investigaciones anteriores (Matsumura-Tundisi et al., 2010; Bustamante et al., 2012; Silva et al., 2012). Esta microalga se caracteriza por ser cosmopolita y tolerar una amplia gama de condiciones ambientales, formando floraciones en sistemas meso- a hipereutróficos, en donde se ha llegado a registrar densidades entre 44175 ind./l y 103329 ind./l en el embalse eutrófico de Embalse Riogrande II Antioquia, Colombia (Bustamante et al., 2012), 28564 ind./l en el embalse mesotrófico de Minas Gerais el Furnas (Silva et al., 2012) y 25000 ind./1 en el reservorio eutrófico de Billings, sureste de Brasil (Matsumura-Tundisi et al., 2010).

En el lago urbano de Lo Méndez, $C$. furcoides mantuvo una alta abundancia durante todo el

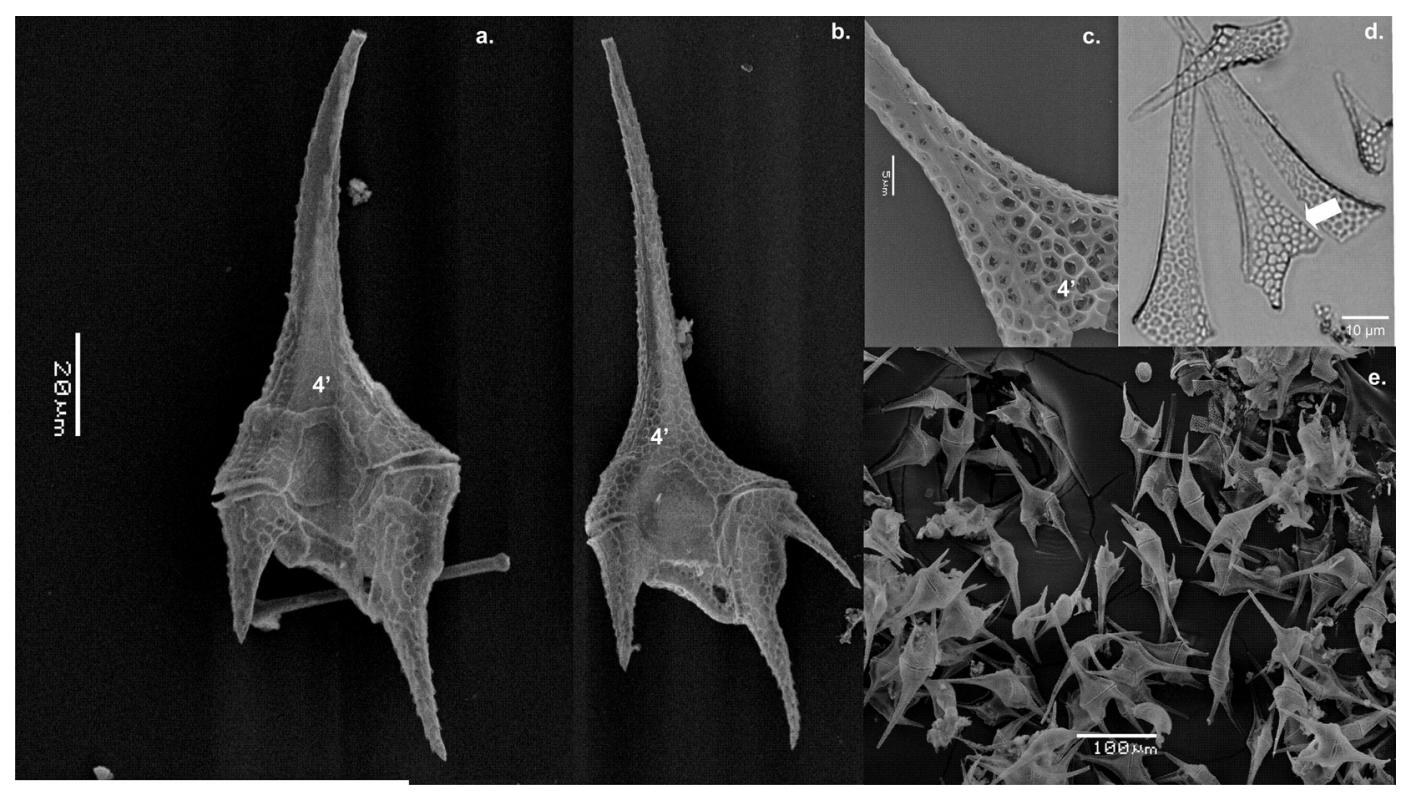

Figura 5. Variabilidad morfológica de $C$. furcoides: a) Vista ventral de $C$. furcoides con dos cuernos en la hipoteca. b) Vista ventral de $C$. furcoides con tres cuernos en la hipoteca. c) Detalle de la epiteca mostrando la 4 placa apical corta (vista ventral). d) Separación de placas con hipoclorito de sodio. e) Floración de $C$. furcoides, SEM. Morphological variability of C. furcoides: a) Ventral view of C. furcoides with two horns on hypotheca. b) Ventral view of C. furcoides with three horns on hypotheca. c) Epitheca detail showing four short apical plate (ventral view). d) Separation of plates with sodium hypochlorite. e) C. furcoides blooms, SEM. 


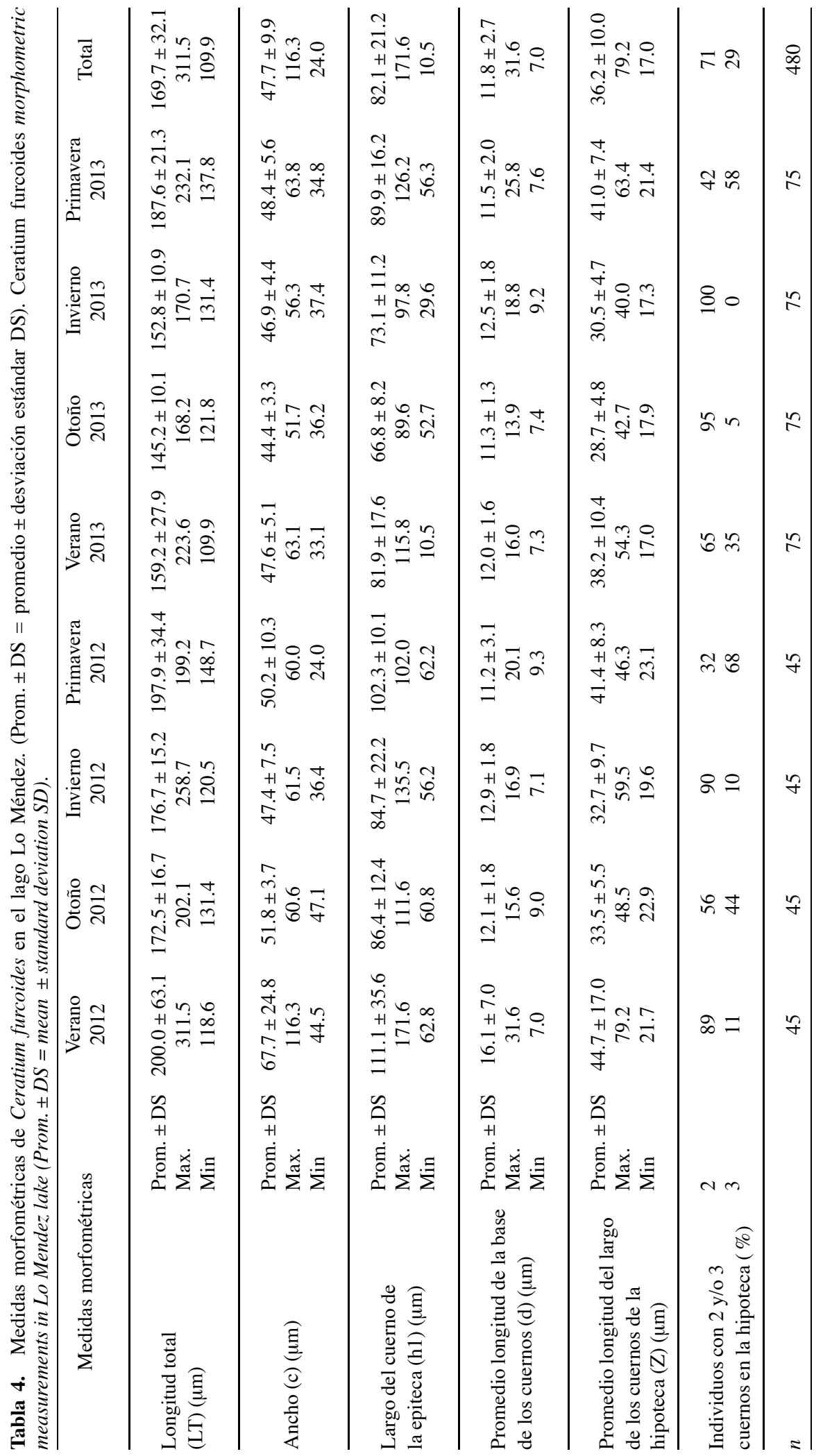


año, que se relacionó principalmente con concentraciones de $\mathrm{NT}, \mathrm{NO}_{3}^{-}$y $\mathrm{NH}_{4}^{+}$, en correspondencia con estudios previos obtenidos en otros lagos de Suramérica, que asocian la presencia de $C$. furcoides principalmente con las concentraciones de $\mathrm{NO}_{3}^{-}$y $\mathrm{NH}_{4}^{+}$(Bustamante et al., 2012), $\mathrm{NO}_{3}^{-} \mathrm{y}$ $\mathrm{NO}_{2}^{-}$(Silva et al., 2012), o NT y PT (MatsumuraTundisi et al., 2010). Se han sugerido otras características limnológicas, como la conductividad eléctrica, concentración de óxido de silicio $\left(\mathrm{SiO}_{2}\right)$, o el aumento en el tiempo de residencia y en la estabilidad de la columna agua, como influyentes en la abundancia de $C$. furcoides (Bustamante et al., 2012; Silva et al., 2012; Meichtry et al., 2014).

La temperatura a la que ocurrieron las floraciones osciló entre 12 y $19{ }^{\circ} \mathrm{C}$, temperaturas inferiores a las reportadas en proliferaciones de lagos tropicales (18-24 ${ }^{\circ} \mathrm{C}$ ) (Bustamante et al., 2012) y acordes con las de desarrollo óptimo para la especie $\left(15-25^{\circ} \mathrm{C}\right)$ (Pollingher, 1988; Ginkel et al., 2001). Durante el otoño, las condiciones ambientales adecuadas para la proliferación del alga fueron asociadas a NT, $\mathrm{NH}_{4}^{+}$y $\mathrm{PO}_{4}^{3-}$ permitiendo el aumento de su abundancia durante dos años consecutivos. Una mayor concentración de $\mathrm{PO}_{4}^{3-} \mathrm{du}-$ rante el otoño del 2012, podría haber determinado el aumento de la densidad de individuos en comparación con el otoño del año siguiente. Las especies del genero Ceratium tienen la capacidad de asimilar fósforo tanto orgánico como inorgánico, lo que podría haber favorecido la mayor densidad de células durante el periodo de mayor concentración de $\mathrm{PO}_{4}^{3-}$ y el inicio de la floración en la época de menor concentración (Ginkel et al., 2001).

Durante la floración se elevó la concentración de la clorofila $a$ producto del aumento de la abundancia del dinoflagelado, produciendo una disminución de la transparencia del agua, así como un aumento en la concentración de oxígeno disuelto debido al incremento en la actividad fotosintética (Lindström, 1992). En general, la gran biomasa de microalgas en una floración genera importantes variaciones diarias de oxígeno, con valores muy altos durante el día y bajos en la noche (Harper, 1992; Ginkel et al., 2001). El posterior decline de la floración causa una alta tasa de descomposición del material acumulado, que es acompañado por el consumo de oxígeno, lo que produce el agotamiento en la concentración local disponible, condiciones de anoxia y subsecuentes mortandades masivas de organismos acuáticos (Wetzel, 2001). En el lago Otario fue descrita la muerte de peces asociada al colapso de una proliferación de $C$. hirundinella (Nicholls et al., 1980). En Lo Méndez, los efectos del decline de la población de $C$. furcoides no fueron evidentes, manteniendo las concentraciones de oxígeno disuelto requeridas para los organismos que allí habitan (la mayor densidad de peces en el lago corresponde a la carpa Cyprinus carpio, reconocida por sobrevivir en mínimas concentraciones de oxígeno).

En lagos ricos en nitrógeno orgánico, individuos del género Ceratium coexisten con algunas especies de cianobacterias que también pueden llegar a generar densas floraciones en este tipo de sistemas, siendo típicos representantes del grupo funcional $\mathrm{L}_{M}$ (Reynolds et al., 2002; Padisák et al., 2009). En el caso de Lo Méndez, este grupo estuvo ausente, no obstante, se presentó una sustitución del grupo M (Microcystis sp.) por el $\mathrm{L}_{O}$ (C. furcoides) en el verano del 2012. El aumento de abundancia de esta cianobacteria coincidió con la mayor concentración de $\mathrm{NH}_{4}^{+}$registrada durante este estudio (en otros meses del año estuvo ausente o presentó muy baja abundancia), lo que pudo favorecer el crecimiento de las cianobacterias, que asimilan más rápidamente el $\mathrm{NH}_{4}^{+}$ que el $\mathrm{NO}_{3}^{-}$como fuente de nitrógeno (Jacoby et al., 2000), y restringir la abundancia del dinoflagelado. La floración de la cianobacteria fue precedida por el dinoflagelado, como ha sido descrito por Reynolds (1997). Otras microalgas, como la diatomea A. formosa (grupo C), típica de pequeños lagos eutróficos, alternaron con las floraciones de $C$. furcoides dominando durante el invierno.

Otras diatomeas, junto con las algas verdes, aumentaron su abundancia relativa en primavera y verano, coincidiendo con la menor densidad del dinoflagelado. Estas algas también se vieron favorecidas por las altas concentraciones de nutrientes en el lago y los cambios estacionales de temperatura. Estudios previos sobre la comuni- 
dad fitoplanctónica en Lo Méndez mostraron que las algas verdes y las diatomeas dominaban la comunidad, que estaba compuesta por 70 taxa de 6 diferentes clases (Cyanobacteria, Dinophyceae, Chrysophyceae, Bacillarophyceae, Euglenophyceae y Chlorophyceae) (Parra et al., 1981). Actualmente la comunidad es más simple, con una menor riqueza y dominada por dinoflagelados y diatomeas.

El proceso de eutrofización del lago favoreció la presencia de algunas especies, como los dinoflagelados, que a su vez podrían determinar cambios en la comunidad. Sin embargo, con la información histórica disponible, se dificulta establecer una relación causal del efecto del dinoflagelado sobre la comunidad.

Las variaciones estacionales en la longitud total y en el número de cuernos de la hipoteca observadas en $C$. furcoides, han sido reportadas previamente para $C$. hirundinella como respuesta morfológica ante los cambios de las condiciones ambientales (Bertolo et al., 2012; Pereira et al., 2013; Varol, 2015). Durante la floración predominaron individuos de menor tamaño y con dos cuernos antapicales, mientras que con una menor abundancia se presentaron individuos con un tercer cuerno en la hipoteca y de mayor tamaño principalmente en primavera. Factores como la concentración de $\mathrm{NH}_{4}^{+}$y $\mathrm{NO}_{2}^{-}$influyen sobre una mayor longitud total del dinoflagelado, en concordancia con observaciones realizadas en $C$. hirundinella por Gligora et al. (2003), quienes determinaron que la concentración de NT influyó en el cambio de la longitud total y el tamaño del cuarto cuerno antapical. La formación de un tercer cuerno antapical facilitaría la flotabilidad de la célula cuando la temperatura es elevada, además de mejorar la protección contra la depredación del zooplancton y de peces planctívoros (Moyá et al., 1984; Hamlaoui et al., 1998; Bertolo, 2010). Durante este periodo también fueron abundantes otras microalgas de las clases Chlorophyceae, Bacillariophyceae y Cryptophyceae, lo que sugiere que podría estar asociado a procesos de competencia interespecífica.

La alta concentración de nutrientes durante el ciclo anual en el lago Lo Méndez propicia la pro- liferación de microalgas que, como $C$. furcoides, se ven beneficiadas ante las condiciones del ecosistema. La plasticidad fenotípica de $C$. furcoides podría favorecer la capacidad invasora de esta alga, proporcionado una ventaja adicional para generar floraciones en el cuerpo de agua, así como para establecerse y dominar en el nuevo hábitat. Esta especie por tanto podría invadir nuevos ambientes con similares condiciones tróficas, por lo que se requiere estudiar la distribución del dinoflagelado sobre todo en cuerpos de agua utilizados para abastecimiento del recurso hídrico. Se recomiendan programas de monitoreo en el lago urbano de Lo Méndez que den cuenta de los cambios del ecosistema y de la abundancia de los dinoflagelados en el contexto del uso recreativo de este sistema y de reservorio de agua.

\section{CONCLUSIONES}

Se describe por primera vez en Chile la ocurrencia de Ceratium furcoides. Su presencia da cuenta de la rápida invasión del dinoflagelado en los ecosistemas acuáticos continentales de Suramérica. La plasticidad fenotípica le permite generar un gran número de individuos de pequeño tamaño y menor ornamentación durante la floración. El resto de año sobreviven individuos de mayor tamaño y más complejos morfológicamente. Los hábitats con alta concentración de nutrientes, como el lago somero de Lo Méndez, benefician su dominancia.

\section{AGRADECIMIENTOS}

Estos datos fueron generados gracias al apoyo de las siguientes fuentes de financiamiento: FONIS SA13I20211 y VRID No 477/12. A la beca CONICYT doctorado nacional No 21130171 y proyecto CRHIAM/Conicyt/Fondap 15130015. Agradecemos también a todo el equipo de muestreo y del laboratorio de Química Ambiental del Centro EULA, Universidad de Concepción. A Enrique Ascencio por sus aportes y comentarios sobre la taxonomía del dinoflagelado. 


\section{BIBLIOGRAFÍA}

ANDRÉS, B. 2012. Lagunas urbanas en Concepción. Una fuente de agua para enfrentar emergencias?.

Revista Aidis, 17-20.

AMERICAN PUBLIC HEALTH ASSOCIATION (APHA), AMERICAN WATER WORKS ASSOCIATION (AWWA) \& WATER POLLUTION CONTROL FEDERATION (WPCF). 2012. Standard methods for the examination of water and wastewater, $22^{\text {nd }}$ ed. APHA, Washington. USA.

BERTOLO, A., G. LACROIX, F. LESCHER-MOUTOUÉ \& J. HJELM. 2010. Relationship between fish and the number of horns in Ceratium hirundinella (Dinophyceae): a food-web-mediated Effect on algal morphology?. Journal of Phycology, 46: 33-40.

BOLTOVSKOY, A. 1989. Thecal morphology of the dinoflagellate Peridinium gutwinskii. Nova Hedwigia, 4(9): 369-380.

BUSTAMANTE, C., J. J. RAMÍREZ, A. BOLTOVSKOY \& A. VALLEJO. 2012. Spatial and temporal change characterization of Ceratium furcoides (Dinophyta) in the equatorial reservoir Riogrande II, Colombia. Acta Limnologica Brasiliensia, 24(2): 207-219.

CALADO, A. J. \& J. LARSEN. 1997. On the identity of the type species of the genus Ceratium Schrank (Dinophyceae), with notes on C. hirundinella. Phycologia, 36: 500-505.

CARTY, S. 2003. Dinoflagellates. In: Freshwater algae of North America, Ecology and classification. J. D. Wehr \& R. G. Sheath (ed.): 685-714. Academic Press, New York.

DONAGH, M. E., M. A. CASCO \& M. C. CLAPS. 2005. Colonization of a Neotropical Reservoir (Córdoba, Argentina) by Ceratium hirundinella (O. F. Müller). International Journal of Limnology, 41(4): 291-299.

GLIGORA, M., A. PLENKOVICC-MORAJ \& I. TERNJEJ. 2003. Seasonal distribution and morphological changes of Ceratium hirundinella in two mediterranean shallow lakes. Hydrobiologia, 506-509: 213-220.

GINKEL, V. C., B. C. HOHLS \& E. VERMAAK. 2001. A Ceratium hirundinella (O.F. Müller) bloom in Hartbeespoort Dam, South Africa. Water SA., 27(2): 269-276.

GINKEL, V. C., C. HONGQING, F. RECKNAGEL \& D. PLESSIS. 2007. Forecasting of dinoflagellate blooms in warm-monomictichypertrophic re- servoirs in South Africa by means ofrule-based agents. Water SA., 33(4).

HAMLAOUI, S., A. COUTÉ, G. LACROIX, F. LESCHER-MOUTOUÉ. 1998. Nutrient and fish effects on the morphology of the dinoflagellate. Eco$\log y, 321: 39-45$.

HARPER, D. 1992. Eutrophication of freshwaters, principles, problems and restoration. Chapman \& Hall. London.

HART, R. C. \& P. D. WRAGG. 2009. Recent blooms of the dinoflagellate Ceratium in Albert Falls Dam (KZN): History, causes, spatial features and impacts on a reservoir ecosystem and its zooplankton. Water S.A., 35(4): 455-468.

HEANEY, S. I., J. W. G. LUND, H. M. CANTER \& K. GRAY. 1988. Population dynamics of Ceratium spp. in three English lakes, 1945-1985. Hydrobiologia, 161: 133-148.

JACOBY, J. M., D. C., COLLIER, E. B. F., WELCH, J., HARDY \& M. CRAYTON. 2000. Environmental factors associated with a toxic bloom of $M i$ crocystis aeruginosa. Canadian Journal of Fisheries and Aquatic Sciences, 57: 231-240.

LINDSTRÖM, K., 1992. Ceratium in Lake Erken: vertical distribution, migration and form variation. Nordic Journal of Botany, 12: 541-556.

MARGALEF, R. 1983. Limnología. Omega. Barcelona. España.

MATSUMURA-TUNDISI, T., J. G. TUNDISI, A. P. LUZIA \& R. M. DEGANI. 2010. Occurrence of Ceratium furcoides (Levander) Langhans 1925 bloom at the Billings Reservoir, São Paulo State, Brazil. Brazilian Journal of Biology, 70(3): 825-829.

MEICHTRY, N., A. BOLTOVSKOY, C. COSTIGLIOLO ROJAS \& R. M. RODRÍGUEZ. 2014. Primer registro del dinoflagelado invasor Ceratium furcoides (Levander) Langhans 1925 en la Argentina y su distribución en el área de influencia del Embalse Yacyretá (río Paraná, Argentina-Paraguay). Limnetica, 33(1): 153-160.

MOYÁ, G. \& G. RAMÓN. 1984. Variación espacio temporal de Ceratium hirundinella, en los embalses de Cuber y Gorg. Limnetica, 1: 285-290.

NICHOLLS, K. M., W. KENNEDY \& C. HANNET. 1980. Fish kill Heart Lake, Otario, associated with the colapse of a masive population of Ceratium hirundinella (Dinophyceae). Freshwater Biology, 10(6): 553-561.

OLIVEIRA, H. S. B., A. N. MOURA \& M. K. CORDEIRO. 2011. First record of Ceratium Schrank, 
1973 (Dinophyceae: Ceratiaceae) in fresh water ecosystems in the semiarid region of Brazil. Check List, 7: 626-628.

PADISÁK, J., L. O. CROSSETTI \& L. NASELLIFLORES. 2009. Use and misuse in the application of the phytoplankton functional classification: a critical review with updates. Hydrobiologia, 621: $1-19$.

PARRA, O. 1998. Una aproximación sistémica para la evaluación de la biodiversidad algal en ambientes acuáticos continentales de Chile. Sociedad Ficológica de América Latina y el Caribe. Sociedad Brasileña de Ficología, 167-178.

PARRA, O. 2006. Estado de conocimiento de las algas dulceacuícolas de Chile (excepto Bacillariophyceae). Gayana, 70(1): 8-15.

PARRA, O., E. UGARTE \& V. DELLAROSSA. 1981. Periodicidad estacional y asociaciones en el fitoplancton de tres cuerpos lenticos en la región de Concepción, Chile. Gayana, 36: 1-35.

PARODI, E. R., N. TROBBIANI \& E. J. CÁCERES. 2007. Cytomorphometric characterization of a population of Ceratium hirundinella fa. austriacum (Dinophyta) during a bloom in a reservoir of the Province of Buenos Aires, Argentina. Algological Studies, 125: 45-55.

PEREIRA, K., J. CONTE, C. C. MÜLLER, K. DORNELLES, J. K. FRIZZO, T. A. VEIGA LUDWIG \& L. S. CARDOSO. 2013. First record of expansive Ceratium Schrank, 1793 species (Dinophyceae) in Southern Brazil, with notes on their dispersive patterns in Brazilian environments. Check List, 9(4): 862-866.

POLLINGHER, U. 1988. Freshwater armored dinoflagellates: growth, reproduction strategies, and population dynamics. In: Growth and reproductive strategies of freshwater phytoplankton. C. Sandgren (ed.): 134-174. Cambridge, University Press. New York.

POPOVSKÝ, J. \& L. A. PFIESTER. 1990. Dinophyceae (Dinoflagellida). In: Süßwasser flora von Mitteleuropa. H. Ettl, J. Gerloff \& H. Heynig (ed.): 1-272. Pektrum Akademischer, Heidelberg.

REYNOLDS, C. S. 1997. Vegetation Processes in the pelagic: a model for ecosystem theory. Ecology Institute. Stuttgart. Germany.

REYNOLDS, C. S., H. VERA, C. KRUK, L. NASELLI-FLORES \& S. MELO. 2002. Towards a functional classification of the freshwater phyto- plankton. Journal of Plankton Research, 24(5): 417-428.

REYNOLDS, C. S. 2006. The ecology of freshwater phytoplankton. Cambridge. England.

SANTOS-WISNIEWSKI, M. J., L. C. SILVA, I. C. LEONE, R. LAUDARES-SILVA \& O. ROCHA. 2007. First record of the occurrence of Ceratium furcoides (Levander) Langhans 1925, and invasive species in the hydroelectricity power plant Furnas Reservoir, MG, Brazil. Brazilian Journal of Biology, 67(4): 791-793.

SILVA, L. C., I. C. LEONE, M. J. SANTOS-WISNIEWSKI, A. C. PERET \& O. ROCHA. 2012. Invasão do dinoflagelado Ceratium furcoides (Levander) Langhans 1925 em um reservatório tropical e sua relação com as variáveis ambientais. Biota Neotropica, 12(2): 93-100.

SILVERIO, M. J., MONTAÑEZ, G. FRA, E. SARACHO, M. ARJONA, M. AMAYA \& B. TRACCANNA. 2009. Variación Poblacional de Ceratium hirundinella (Dinophyceae) en Embalses Eutróficos de Catamarca (Argentina) y su relación con parámetros ambientales. Huayllu-Bios, 3: 13-31.

SUKENIK, A., O. HADAS, A. KAPLAN \& A. QUESADA. 2012. Invasion of nostocales (Cyanobacteria) to subtropical and temperate freshwater lakes physiological, regional, and global driving forces. Frontiers in Microbiology, 3: 86.

THOMASSON, K. 1963. Araucanian Lakes. Acta Phytogeographica Suecica, 47: 1-139.

UTERMÖHL, H. 1958. Zur vervollkommnung der quantitativen phytoplankton methodik. Mitteilungen Internationale Vereinigung für Theoretische und Angewandte Limnologie, 9: 1-38.

VADRUCCI, M. R., M. CABRINI \& A. BASSET. 2007. Biovolume determination of phytoplankton guilds in transitional water ecosystems of Mediterranean Ecoregion. Transitional Waters Bulletin, 2: 83-102.

VOLLENWEIDER, R. A. \& J. KEREKES. 1982. Eutrophication of waters. Monitoring, assessment and control. OECD, Paris.

VAROL, M. 2015. External morphological variations and temporal distribution of the dinoflagellate $\mathrm{Ce}$ ratium hirundinella in two dam reservoirs in the Tigris River basin (Turkey). Turkish Journal of Botany, 39: 1-5.

WETZEL, R. G. 2001. Limnology: Lake and River Ecosystems. (3'd ed.). Academic Press, San Diego. 
